American J. of Engineering and Applied Sciences 4 (4): 513-519, 2011

ISSN 1941-7020

(C) 2014 S. Rajbhandari et al., This open access article is distributed under a Creative Commons Attribution

(CC-BY) 3.0 license

\title{
The Performance of a Dual Header Pulse Interval Modulation in the Presence of Artificial Light Interferences in an Indoor Optical Wireless Communications Channel with Wavelet Denoising
}

\author{
${ }^{1,2}$ Rajbhandari, S., ${ }^{1}$ Z. Ghassemlooy and ${ }^{2}$ M. Angelova \\ ${ }^{1}$ Optical Communications Research Group, \\ ${ }^{2}$ Intelligent Modelling Lab, \\ School of CEIS, Northumbria University, UK
}

\begin{abstract}
Problem statement: Similar to other baseband modulation schemes, the performance of the Dual Header Pulse Interval Modulation (DH-PIM) is adversely affected by Artificial Light Interferences (ALI) in an indoor Optical Wireless Communications (OWC) channel. Approach: The Discrete Wavelet Transform (DWT) based denoising is studied for reducing the effect of the ALI. Computer simulation is carried out to demonstrate the effectiveness of the proposed system. The Baseline Wander (BLW) affects for a range of bit resolutions is also analyzed. Results: The normalized optical power requirement in the presence of ALI is high in the range of 9-15 dB depending upon the bit resolution. A significant reduction in the optical power penalty is observed with the DWT denoising scheme. Conclusion: The DWT based denoising scheme is effective in reducing the consequence of the ALI. The optical power penalty decreases with an increase in the data rate and the bit resolution, which is due to reduction of the DC values and low frequency spectrum.
\end{abstract}

Key words: Optical wireless communication, digital modulation schemes, artificial light interference, discrete wavelet transform, dual header pulse interval modulation

\section{INTRODUCTION}

The rapid growth of portable devices and information terminals within indoor environment and the high bandwidth demand for each terminal due to the video and data sharing has already enforced the deployment of advanced communication systems using optical wavelengths (Mohamed and Hranilovic, 2009; Ntogari et al., 2009). The radio frequency applications are limited due to the scarcity in available bandwidth as well as a high licensing cost, whereas in the optical domain the huge unlicensed bandwidth, in excess of $200 \mathrm{THz}$, opens up many new opportunities in a large number of bandwidth hungry applications. Moreover, due to confinement of the optical beam within close walls, the same wavelength (or a range of wavelengths) could be reused within the same room, neighbouring rooms and the buildings without any inter-channel and adjacent channel interference. With the optical spectrum is free from Electromagnetic Interference (EMI), the application of the OWC is more suitable for environments where sensitive equipments are needed to be protected from the EMI.

The visible and Infrared (IR) spectrum has been proposed for indoor OWC systems. The clear advantage of the visible light over IR communications is possibilities of dual purposes for room lightening and communications. However, the bandwidth of the visible Light Emitting Diode (LED) is limited to a few $\mathrm{MHz}$ and hence the advanced technologies such as preequalization and optical filtering are necessary to obtain higher data rates (Minh et al., 2009). The uplink is also problematic in visible light communications and mostly relies on the IR for the uplink channel. The OWC system using the IR spectrum has found applications in both the indoor and outdoor environment. The laser source is the most preferable in the outdoor environment and link lengths greater than $6 \mathrm{~km}$ with a data rate greater than $1 \mathrm{Gbps}$ has already been reported (Ciaramella et al., 2009). In the indoor environment, the LED source with a wide beam divergence would be preferable in order to meet the eye-safety standard and also to avoid necessity for tracking.

\section{MATERIALS AND METHODS}

A number of digital modulation schemes had been studied for indoor OWC (Ghassemlooy et al., 2007; Fan et al., 2010). The DHPIM modulation scheme offers a trade-off between bandwidth and power 
efficiencies. The power efficiency of DH-PIM is higher than of On and Off Keying (OOK) and lower than of Pulse Position Modulation (PPM) schemes. On the other hand, DH-PIM is more bandwidth efficient than PPM. Unlike PPM, DH-PIM does not require symbol synchronization, thus greatly simplifying the symbol recovery circuitry at the receiver.

The availability of cheap LEDs and photodiodes in the range of $800-900 \mathrm{~nm}$ makes this wavelength the most suitable for indoor applications. However, the performance of the OWC link at this wavelength is severely affected by the ALI. Unlike the other form of noise like thermal noise and shot noise, which are of Gaussian type, the ALI is non-Gaussian in nature and therefore the matched filter based receiver is not the optimum option. The high pass filtering in conjunction with the matched filter is applied to alleviate the effect of the ALI. In previous studies, a DWT based receiver was proposed to reduce the effect of ALI for OOK modulation schemes (Rajbhandari et al., 2009; 2011). It was shown that the DWT offered an improved performance compared to the high pass filtering with reduced complexity for indoor OWC. In this study, the DWT is applied to the DH-PIM scheme in order to evaluate it effectiveness. The DWT based denoising finds its application in diverse field including medicine, engineering and physics (Ho et al., 2003).

Dual header pulse interval modulation: The DH-PIM scheme belongs to the digital anisochronous pulse modulation scheme in which the information is encoded between the adjacent pulses. Unlike Pulse Interval Modulation (PIM) where symbols start with a pulse, in DH-PIM symbols are initiated with a header of one or two time slot pulse durations. Introducing different header (in this case two headers) further reduces the overall symbol length and hence increases the system throughput. L-DH-PIM ${ }_{\alpha}$ encodes an $\mathrm{M}\left(=\log _{2} \mathrm{~L}\right)$ bits input binary word into a DH-PIM symbol which has a header of $(\alpha+1) \mathrm{T}_{\mathrm{s}}$ duration followed by $\mathrm{d}$-slot representing the information $\mathrm{m}(\mathrm{t})$, where $\alpha$ and $\mathrm{d}$ are positive integers and Ts is the slot duration. The header pattern depends on the Most Significant Bit (MSB) of $\mathrm{m}(\mathrm{t})$. Headers $\mathrm{H}_{0}$ and $\mathrm{H}_{1}$ correspond to the MSB of " 0 " and "1", respectively. The header consists of a pulse of duration $0.5 \alpha \mathrm{T}_{\mathrm{s}}$ and $\alpha \mathrm{T}_{\mathrm{s}}$ for $\mathrm{H}_{0}$ and $\mathrm{H}_{1}$, respectively followed by a guard band of appropriate length. The number of information slots (d) that follows headers is equal to the decimal value of the input word for $\mathrm{MSB}=0$ and the 1's complement of the decimal value of the input word otherwise. The minimum and the maximum length of the symbols are $(\alpha+1)$ and $\left(2^{\mathrm{M}-1}+\alpha\right)$, respectively. Hence the average symbol length and the slot duration for DH-PIM are given by Eq. 1 and 2, respectively:

$$
\overline{\mathrm{L}}=\frac{\left(2^{\mathrm{M}-1}+2 \alpha+1\right)}{2}
$$

$\mathrm{T}_{\mathrm{s}}=\frac{\mathrm{M}}{\overline{\mathrm{L}} \mathrm{R}_{\mathrm{b}}}$

where, $R_{b}$ is the bit rate.

Thus, the DH-PIM pulse train can be represented by Eq. 3 (Aldibbiat, 2001):

$$
\mathrm{x}(\mathrm{t})=\mathrm{I}_{\mathrm{p}} \sum_{\mathrm{k}=0}^{\infty}\left\{\operatorname{rect}\left[\frac{2\left(\mathrm{t}-\mathrm{T}_{\mathrm{k}}\right)}{\alpha \mathrm{T}_{\mathrm{s}}} \mid-\frac{1}{2}\right]+\mathrm{H}_{\mathrm{n}} \operatorname{rect}\left[\frac{2\left(\mathrm{t}-\mathrm{T}_{\mathrm{k}}\right)}{\alpha \mathrm{T}_{\mathrm{s}}} \mid-\frac{3}{2}\right]\right.
$$

where, $I_{p}$ is the peak photocurrent, $n=0,1$ and the rectangular pulse function is defined as Eq. 4:

$\operatorname{rect}[t]=\left\{\begin{array}{rr}1 ;-0.5<t<0.5 \\ 0 \quad \text { otherwise }\end{array}\right.$

Assuming Identical and Independent Distribution (IID) of the input information bits, the Slot Error Rate (SER) of the DH-PIM in a non-dispersive white Gaussian channel with matched filtering is given by Eq. 5 (Aldibbiat, 2001):

$\mathrm{P}_{\mathrm{se}}=\frac{1}{4 \overline{\mathrm{L}}}\left[\begin{array}{c}(4 \overline{\mathrm{L}}=3 \alpha) \mathrm{Q}\left(\frac{\mu \mathrm{kRP} \text { avg }}{\sqrt{\mathrm{N}_{0} \mathrm{R}_{\mathrm{b}}}}\right) \\ +3 \alpha \mathrm{Q}\left(\frac{\mu(1-\mathrm{K}) \mathrm{RP}_{\text {avg }}}{\sqrt{\mathrm{N}_{0} \mathrm{R}_{\mathrm{b}}}}\right)\end{array}\right]$

Where:

$\mathrm{R}=$ The photodetector sensitivity, $\mu=\sqrt{32 \mathrm{M} \overline{\mathrm{L}} 9 \alpha^{2}}$

$\mathrm{k}=$ The threshold level

$\mathrm{P}_{\mathrm{avg}}=$ The averagereceived optical power and $\mathrm{Q}(\mathrm{x})=\frac{1}{2 \pi} \int_{\mathrm{X}}^{\infty} \exp \left(-\frac{\mathrm{u}^{2}}{2}\right) \mathrm{du}$ Throughout this study,

DH-PIM will be referred to as L-DH-PIM ${ }_{\alpha}$, where $\mathrm{L}=2^{\mathrm{M}}$

For example, 16-DH-PIM 2 means a DH-PIM symbol with $\mathrm{M}=4$ and $\alpha=2$.

Flourescent light interference and wavelet denoising: The effect of the ALI for an indoor OWC channel is well studied and has been reported in (Moreira et al., 1997; Narasimhan et al., 1996). Among a number of artificial light sources, the interference produced by fluorescent lamps driven by electronic 
ballasts is the most severe as the Power Spectral Density (PSD) of the interfering signal extends up to 1 $\mathrm{MHz}$. This imposes a significant problem to the baseband modulation scheme as the modulated signal has significant spectrum contents near the DC region of the spectrum. In order to reduce the interfering signal, an electrical High Pass Filter (HPF) can be encompassed at the receiver. However, high pass filtering leads to another problem, commonly known as the Baseline Wander (BLW) effect, due to the variation in the average level of the signal being transmitted. In order to overcome this problem and improve the system performance, a denoising scheme based on the "wavelet transform" has been proposed; which offers similar or better performance to the electrical HPF but with much reduced complexity (Rajbhandari et al., 2009).

In this study, we have adopted the DWT for denoising for the DH-PIM scheme as shown in Fig. 1. The M-bit binary input data $\mathrm{a}_{1 . . \mathrm{M}}$ is first converted to a DH-PIM sequence by the DH-PIM encoder followed by transmitter filter $\mathrm{p}(\mathrm{t})$ with a rectangular pulse duration $\mathrm{T}_{\mathrm{s}}$. DH-PIM pulse trains directly modulate the intensity of the laser diode/LED with the appropriate optical power provided by the biasing circuitry. The receiver front-end consists of a photodiode followed by a Transimpendence Amplifier (TIA). The photocurrent $I_{p}$ generated by the photodiode is the summation of currents due to the modulating signal $\mathrm{I}(\mathrm{t})$ and the interfering signal $\mathrm{mfl}(\mathrm{t})$. The post-detection filter $\mathrm{r}(\mathrm{t})$ is matched to the transmitter filter $\mathrm{p}(\mathrm{t})$. The output of the matched filter is sampled at the $\mathrm{T}_{\mathrm{s}}^{-1}$ rate followed by wavelet denoising and threshold detection modules. In order to simplify the analysis here, the TIA is ignored in the following discussion.

The sampled matched filter output due to the modulating and interfering signals at the receiver are given by Eq. 6 and 7, respectively:

$\mathrm{i}_{\mathrm{K}}=\left.\mathrm{RI}_{\mathrm{px}}(\mathrm{t}) \otimes \mathrm{r}(\mathrm{t})\right|_{\mathrm{t}=\mathrm{KTS}}$

$\mathrm{m}_{\mathrm{k}}=\left.\mathrm{m}_{\mathrm{fI}}(\mathrm{t}) \otimes \mathrm{r}(\mathrm{t})\right|_{\mathrm{t}=\mathrm{KTS}}$

where, $\otimes$ is the convolution operation.

In the absence of noise and interference, the peak output of the matched filter when a "one" is transmittedis is $\sqrt{\mathrm{E}_{\mathrm{p}}}=\left(4 \mathrm{RP}_{\mathrm{avg}} \overline{\mathrm{L}} \sqrt{\mathrm{T}_{\mathrm{s}}}\right) / 3 \alpha$. When a "zero" is transmitted, the peak output is 0 . Considering the threshold level of the threshold detector set at $0.5\left(\mathrm{E}_{\mathrm{p}}\right)^{0.5}$, Eq. 5 can be simplified to Eq. 8:

$$
P_{s e}=Q\left(\sqrt{\frac{E_{p}}{2 N_{0}}}\right)
$$

However, the FLI is a periodic and hence the error probability can be calculated by averaging the slot error probability over a cycle. By considering every slot over a $20 \mathrm{msec}$ interval (i.e., one complete cycle of $\mathrm{m}_{\mathrm{fl}}(\mathrm{t})$ ) and averaging, the SER can be approximated as given in Eq. 9 (Narasimhan et al., 1996):

$$
\mathrm{P}_{\mathrm{se}}=\frac{1}{\mathrm{~N}} \sum_{\mathrm{k}=1}^{\mathrm{N}} \frac{1}{4 \overline{\mathrm{L}}}\left[\begin{array}{r}
(4 \overline{\mathrm{L}}-3 \alpha) \mathrm{Q}\left(\frac{\sqrt{\mathrm{E}_{\mathrm{p}} 2+\mathrm{mk}}}{\sqrt{\mathrm{N}_{0} 2}}\right) \\
+3 \alpha \mathrm{Q}\left(\frac{\sqrt{\mathrm{E}_{\mathrm{p}} 2+\mathrm{mk}}}{\sqrt{\mathrm{N}_{0} 2}}\right)
\end{array}\right]
$$

where, $\mathrm{N}$ is the total number of DH-PIM slots over a $20 \mathrm{~ms}$.

FLI is the dominant source of noise in indoor environment with Optical Power Penalty (OPP) as high as $10 \mathrm{~dB}$ or more depending of course on the data rate. In order to lessen the effect of FLI, HPF or DWT can be adopted. In the latter the received signal is decomposed into a number of DWT levels corresponding to different bands of frequencies, which also includes the interference band. The band of frequency corresponding to the interference is then readily removed and the signal is easily reconstructed. Consider a DWT composed of lowpass $h(n)$ and highpass $\mathrm{g}(\mathrm{n})$ filters. The DWT involves splitting the signal $\mathrm{y}(\mathrm{n})$ into lowpass $\mathrm{y}_{11}$ and highpass $\mathrm{y}_{1 \mathrm{~h}}$ signals as given by Eq. 10 and 11; respectively (Burrus et al., 1998):

$$
\mathrm{y}_{1 \mathrm{n}}(\mathrm{k})=\sum_{\mathrm{n}} \mathrm{y}(\mathrm{n}) \mathrm{g}(2 \mathrm{k}-\mathrm{n})
$$

$$
\mathrm{y}_{\mathrm{II}}(\mathrm{k})=\sum_{\mathrm{n}} \mathrm{y}(\mathrm{n}) \mathrm{h}(2 \mathrm{k}-\mathrm{n})
$$

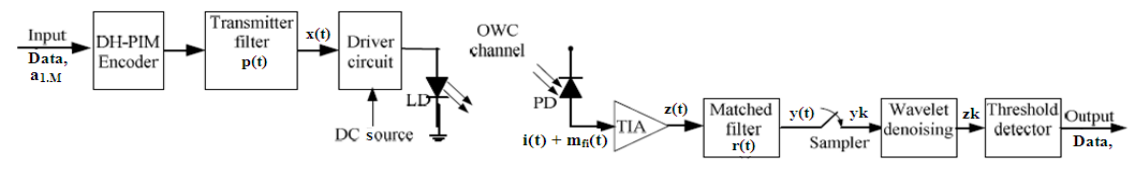

Fig. 1: A schematic diagram of OWC system employing DWT at the receiver 
Am. J. Engg. \& Applied Sci., 4 (4): 513-519, 2011
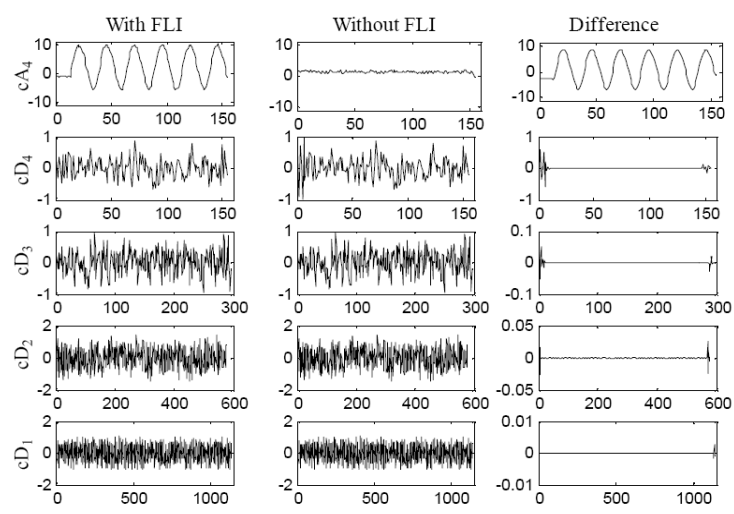

Fig. 2: 4-level DWT decomposition of the 8-DH-PIM 2 received signal with and without FLI

The approximation coefficient $\mathrm{y}_{11}$ is further decomposed into different DWT coefficient levels until modulating and interfering signals are effectively detached.

In order to illustrate the concept of the DWT denoising for removing FLI, details and approximation coefficients of a 4-level decomposition of received 8$\mathrm{DH}_{-} \mathrm{PIM}_{2}$ signal at a data rate of $20 \mathrm{Mbit} / \mathrm{s}$ with and without FLI using the Daubechies wavelet $(\mathrm{db} 8)$ is shown in Fig. 2 (first and second column respectively). The interference photocurrent $\mathrm{m}_{\mathrm{fI}}(\mathrm{t})$ is arbitrarily made four times that of the signal photocurrent $i(t)$ and the Additive White Gaussian Noise (AWGN) is ignored. The difference between DWT coefficients is shown in the third column. The differences in the detail coefficients at all levels are negligible, however the significant difference in the approximation (cA4) is noticeable. The approximation coefficient corresponds to the frequency band from DC to $1.25 \mathrm{MHz}$. Hence, in principle, the proposed denoising can be achieved by means of optimal decomposition and diminution of the coefficients corresponding to the interference signal. The decomposition level is calculated using Eq. 12:

$\gamma=-\left\lfloor\log _{2}\left(\frac{\mathrm{MF}_{\mathrm{c}}}{\mathrm{LR}_{\mathrm{b}}}\right)\right\rfloor$

Where:

[. $\rfloor=$ The floor function

$\mathrm{F}_{\mathrm{c}}=$ The desired cut-off frequency

A cut-off frequency of $0.5 \mathrm{MHz}$ is incorporated in this study because previous study has been shown that this frequency is optimal (Dickenson and Ghassemlooy, 2005).

\section{RESULTS AND DISCUSSION}

For the comparative studies of the performance of the OWC system with and without the DWT denoising module, the NOPR (normalized to OOK at $1 \mathrm{Mbit} / \mathrm{s}$ ) to achieve an error probability of $10^{-6}$ is calculated under three different conditions: (1) a channel without interference, (2) a channel with interference and (3) a channel with interference and with DWT. The channel is assumed to be a Line Of Sight (LOS) with the AWGN. The FLI is modelled according to (Moreira et al., 1997), with a switching frequency of $37.5 \mathrm{KHz}$. Since the DC component is filtered by DWT, the threshold level set midway between " 1 " and " 0 " levels, in the absence of any BLW and is given as Eq. 13 (Hayes et al., 2000):

$$
\mathrm{k}=\frac{\left(4 \mathrm{RP}_{\text {avg }} \overline{\mathrm{L}} \sqrt{\mathrm{T}_{\mathrm{s}}}\right)}{3 \alpha}\left(\frac{1}{2}-\frac{3 \alpha}{4 \overline{\mathrm{L}}}\right)
$$

The NOPR versus the data rates for 4, 8, 16 and 32 $\mathrm{DH}^{\mathrm{PIM}} \mathrm{M}_{1}$ with and without the DWT denoising in the presence of the FLI for a SER of $10^{-6}$ is given in Fig. 3. Also shown is NOPR in a Gaussian channel. Similar to OOK (Rajbhandari et al., 2009), DH-PIM requires an almost constant optical power to achieve a SER of $10^{-6}$

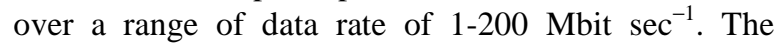
optical power penalty tends to fall as the data rate increases. The power penalties for $\mathrm{DH}-\mathrm{PIM}_{1}$ at $1 \mathrm{Mbit}$ $\sec ^{-1}$ with FLI without FLI Difference cD1 cD2 cD3 cD4 cA4 is $15.3-15.1 \mathrm{~dB}$ for $\mathrm{L}=4$ and 32 , falling to approximately 5-6 at $200 \mathrm{Mbit}^{-1} \mathrm{sec}^{-1}$. With DWT we observe a significant reduction in the optical power requirements for all orders of $\mathrm{DH}-\mathrm{PIM}_{1}$ at data rates above 10 Mbit $\mathrm{sec}^{-1}$. Due to a relatively high normalized cut-off frequency (normalized to the data rate), very little or no improvement can be achieved using a DWT at $1 \mathrm{Mbit} \mathrm{sec}^{-1}$. The general trend shows that the DWT transform is more effective tool at higher data rates and higher bit resolutions. There is a progressive reduction in the power penalty as the bit resolution increases from $2-5$, which is due to the reduction in DC and low frequency components (Aldibbiat, 2001).

The optical power penalty for DH-PIM 1 at $10 \mathrm{Mbit} / \mathrm{s}$ is $\sim 2.47 \mathrm{~dB}$ for $\mathrm{L}=4$, which reduces to $\sim 0.3 \mathrm{~dB}$ for $\mathrm{L}=$

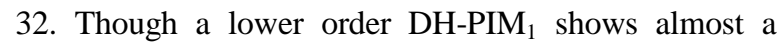
constant power penalty beyond the data rate of $20 \mathrm{Mbit} / \mathrm{s}$ $(\sim 1.8 \mathrm{~dB}, \sim 1.2 \mathrm{~dB}$ and $\sim 0.7 \mathrm{~dB}$ for $\mathrm{L}=4,8$ and 16$)$, the power penalty is completely eliminated for $\mathrm{L}=32$. 
Am. J. Engg. \& Applied Sci., 4 (4): 513-519, 2011

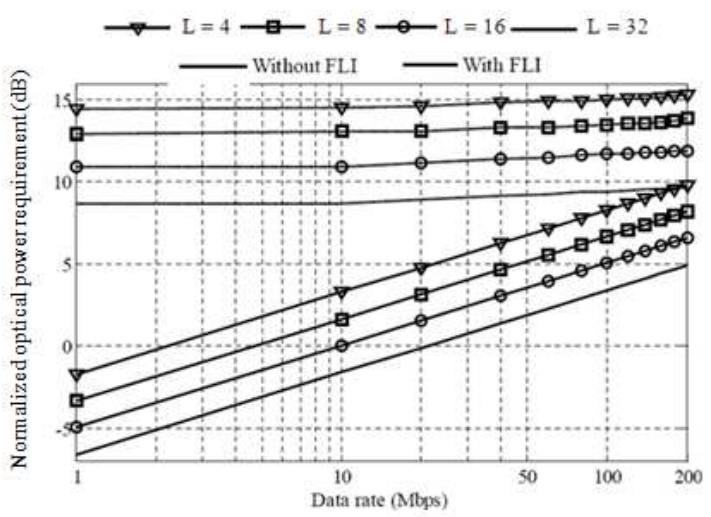

(a)

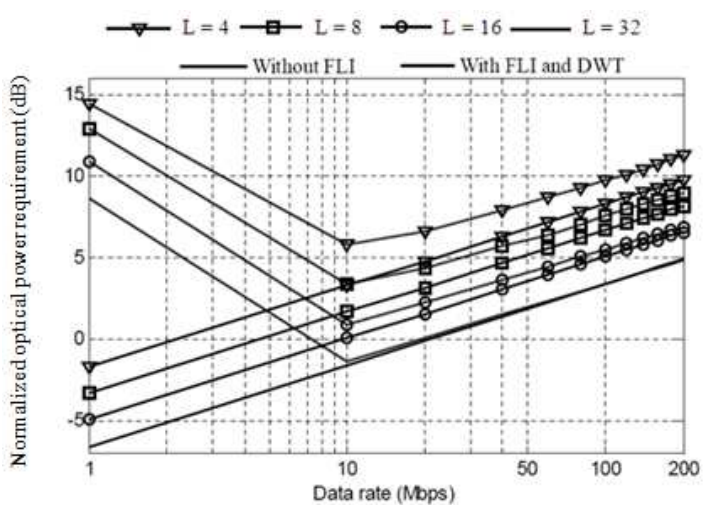

(b)

Fig. 3: The NOPR versus the data rates for 4, 8, 16 and 32 DH-PIM $_{1}$ with and without the FLI: (a) without DWT denoising and (b) with DWT denoising

The NOPR performance against the data rates for 4, 8, 16 and 32 DH-PIM ${ }_{2}$ with and without DWT in the presence of the FLI and for a SER of $10^{-6}$ is given in Fig. 4. The general behaviour of DH-PIM 2 is similar to that of DH-PIM1 for both with and without DWT. However, the optical power penalties without DWT are

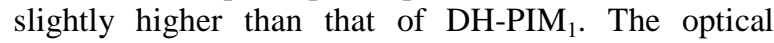
power penalties decrease from $\sim 16.1-15.3 \mathrm{~dB}$ and $~$ 5.6-4.7 dB for $\mathrm{L}=4$ and 32 at 1 and $200 \mathrm{Mbit} / \mathrm{s}$, respectively. Compared to the system without DWT, we have observe $\sim 10 \mathrm{~dB}$ and $\sim 9.7 \mathrm{~dB}$ reduction in optical power penalty at $10 \mathrm{Mbit} / \mathrm{s}$ for $\mathrm{L}=4$ and 32 , respectively. However, at $200 \mathrm{Mbit} / \mathrm{s}$ data rate, the optical power penalties reduces to $\sim 1.2 \mathrm{~dB}, \sim 0.6 \mathrm{~dB}, \sim$ $0.4 \mathrm{~dB}$ and $\sim 0 \mathrm{~dB}$ from $\mathrm{L}=4,8,16$ and 32 , respectively. It should be noted that the power penalty experience with the system employing DWT based denoising are not due to the ineffectiveness of the DWT to reduce the FLI.

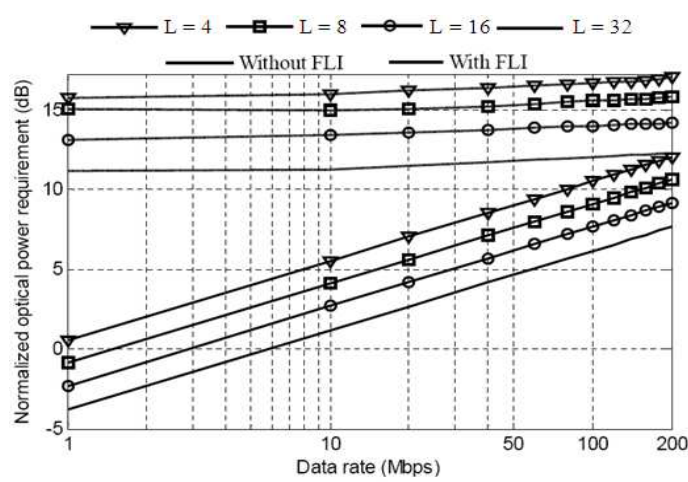

(a)

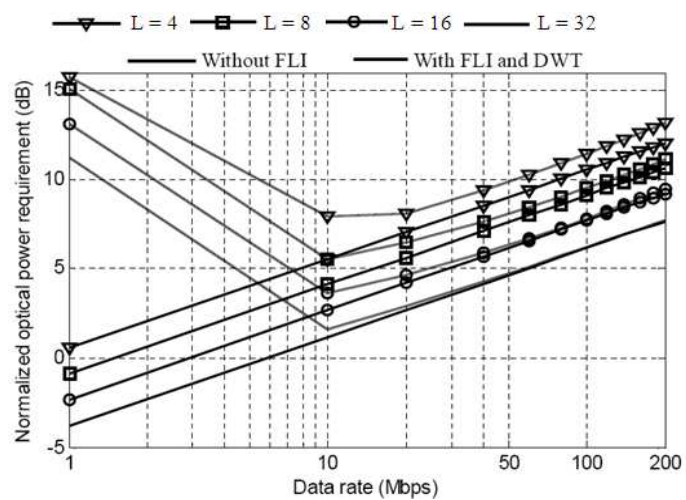

(b)

Fig. 4: The NOPR versus the data rates for 4, 8, 16 and 32 DH-PIM $_{1}$ with and without the FLI: (a) without DWT denoising and (b) with DWT denoising

In fact, it is due to the BLW interference introduced by the DWT itself. Intuitively from the above discussion, it is expected that the BLW effect reduces as the bit resolution increases. In order to analyse the effect of BLW, the difference between the expected matched filter output (without DWT) and the DWT output at a normalized cut-off frequency of 0.1 for 8-DHPIM1\&2 at a data rate of $10 \mathrm{Mbit} / \mathrm{s}$ is shown in Fig. 5. Note that the maximum frequency is normalized to one. The probability distribution of the baseline wander in systems employing DH-PIM is clearly non-Gaussian as the input data streams are not IID. Comparing the two histograms, it is evident that the variance of DH-PIM1 is marginally smaller than that of DH-PIM2. This is due to the fact that the DC component of DH-PIM ${ }_{2}$ is more

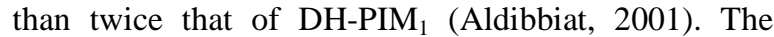
higher order of DH-PIM offers more resilience to the BLW effect as the variance decreases from 0.24060.0931 and from $0.2396-0.1721$ as the bit resolution increases from 2-5 for DH-PIM1 and 2, respectively. 
Am. J. Engg. \& Applied Sci., 4 (4): 513-519, 2011

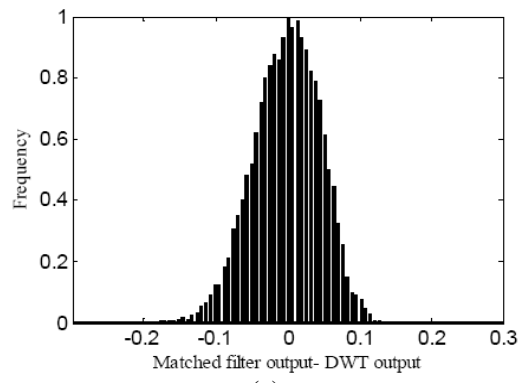

(a)

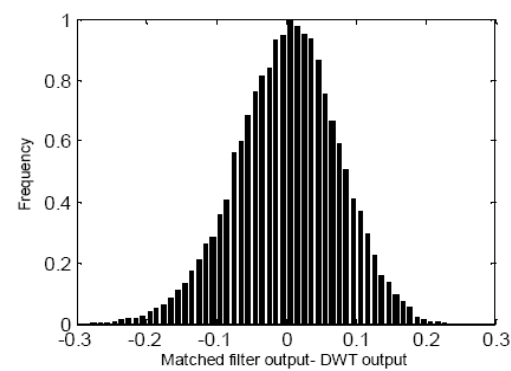

(b)

Fig. 5: The histograms of the matched filter output for 8-DH-PIM with a normalized cut-off frequency of 0.1

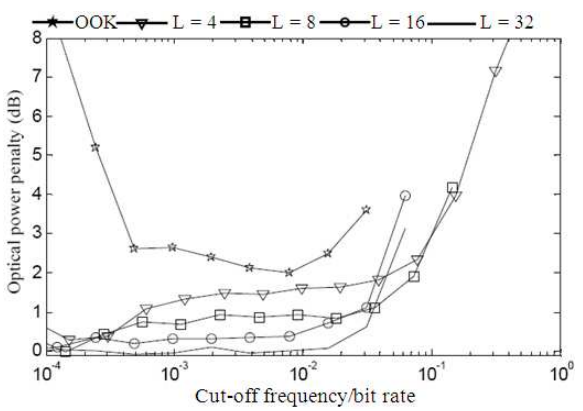

(a)

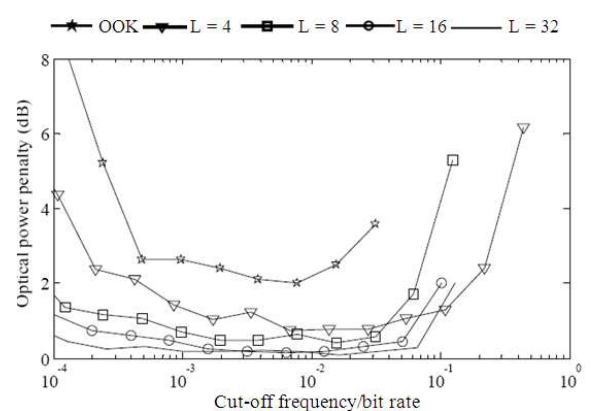

(b)

Fig. 6: The optical power penalty versus the normalized cut-on frequency for $\mathrm{L}=4,8,16,32$ at $10 \mathrm{Mbit}$ $\sec ^{-1}$ : (a) DH-PIM 1 and (b) DH-PIM
In order to show the overall effect of BLW at different cut-off frequencies, the optical power penalty at different normalized cut-off frequencies at a data rate of $10 \mathrm{Mbit} / \mathrm{s}$ is shown in Fig. 6 for DH-PIM ${ }_{1 \& 2}$ with $\mathrm{L}=$ 4-32. Also shown is the BLW induced optical power penalty for OOK at the same data rate. It is apparent from the figure that the effect of the BLW in DHPIM is significantly lower than that of OOK irrespective of the bit resolution and $\alpha$. It is also noticeable that the power penalty decreases as the bit resolution increases. This is due to progressive decrease in the DC content as the bit resolution increases. There is evidently a window of cut-off frequencies where the BLW level is minimum. The DH-PIM scheme shows a wide cut-off frequency window, thus resulting in more flexibility when designing a filter. The window of the cut-off frequencies depends on the bit resolution and $\alpha$. It is relatively large for $\alpha=1$ compared to $\alpha=2$. Figure 6 also demonstrates that the optical power penalty becomes negligible for $\mathrm{L}=32$ for both $\alpha=1$ and 2, which justifies the null optical power penalty for 32DH-PIM as in Fig. 3 and 4.

\section{CONCLUSION}

The performance of DWT based denoising for DHPIM schemes in an indoor OWC in the presence of the FLI is reported here. The higher order of DH-PIM shows more resilience to the FLI; however the power penalty without denoising can be higher than $10 \mathrm{~dB}$, depending on the data rates. The DWT based denoising is effective in reducing the consequence of the FLI and a significant optical power penalty reduction $(\sim 10 \mathrm{~dB}$ at $10 \mathrm{Mbps})$ is observed. The optical power penalty decreases with an increase in the data rate and bit resolution, which is due to reduction of the DC values and low frequency spectrum. The study shows that DWT introduces another form of interference known as baseline wander. The baseline wander effect reduces as the bit resolution increases, providing evidence of the null power penalties at the bit resolution of five.

\section{REFERENCES}

Aldibbiat, N.M., 2001. Optical wireless communication systems employing dual header pulse interval modulation (DH-PIM). Ph.D Thesis, Sheffield Hallam University.

Burrus, C.S., R.A. Gopinath and H. Guo, 1998. Introduction to Wavelets and Wavelet Transforms. 1st Edn., Prentice Hall, Upper Saddle River, NJ., ISBN-10: 0134896009, pp: 268. 
Ciaramella, E., Y. Arimoto, G. Contestabile, M. Presi and A. D'Errico et al., 2009. 1.28 terabit/s (32x40 Gbit/s) wdm transmission system for free space optical communications. IEEE J. Selected Areas Commun., 27: 1639-1645. DOI: 10.1109/JSAC.2009.091213

Dickenson, R.J. and Z. Ghassemlooy, 2005. WaveletAI equalization and detection for indoor diffuse infrared wireless systems. Int. J. Commun. Syst., 18: 247-266. DOI: $10.1002 /$ dac. 702

Fan, Y., B. Bai and R.J. Green, 2010. PPMPWM: A new modulation format for wireless optical communications. Proceedings of the 7 th International Symposium on Communication Systems Networks and Digital Signal Processing, Jul. 21-23, IEEE Xplore Press, Newcastle upon Tyne, pp: 604-609.

Ghassemlooy, Z., W.O. Popoola, S. Rajbhandari, M. Amiri and S. Hashemi, 2007. A synopsis of modulation techniques for wireless infrared communication. Proceedings of the ICTON Mediterranean Winter Conference, Dec. 6-8, IEEE Xplore Press, Sousse, pp: 1-6. DOI: 10.1109/ICTONMW.2007.4446952

Hayes, A.R., Z. Ghassemlooy, N.L. Seed and R. McLaughlin, 2000. Baseline-wander effects on systems employing digital pulse-interval modulation. IEE Optoelectronics, 147: 295-300. DOI: 10.1049/ip-opt:20000565

Ho, C.Y.F., B.W.K. Ling, T.P.L. Wong, A.Y.P. Chan and P.K.S. Tam, 2003. Fuzzy multiwavelet denoising on ECG signal. Elect. Lett., 39: 11631164. DOI: $10.1049 / \mathrm{el}: 20030757$
Minh, H.L., D. O'Brien, G. Faulkner, L. Zeng and K. Kyungwoo et al., 2009. 100-Mb/s NRZ visible light communications using a postequalized white LED. IEEE Photonics Technol. Lett., 21: 1063-1065. DOI: 10.1109/LPT.2009.2022413

Mohamed, M.D.A. and S. Hranilovic, 2009. Optical impulse modulation for indoor diffuse wireless communications. IEEE Trans. Commun., 57: 499-508. DOI: 10.1109/TCOMM.2009.02.070293

Moreira, A.J.C., R.T. Valadas and A.M.D.O. Duarte, 1997. Optical interference produced by artificial light. J. Wireless Netw., 3: 131-140. DOI: 10.1023/A:1019140814049

Narasimhan, R., M.D. Audeh and J.M. Kahn, 1996. Effect of electronic-ballast fluorescent lighting on wireless infrared links. IEE Optoelectronics, 143: 347-354. DOI: 10.1049/ip-opt:19960877

Ntogari, G., T. Kamalakis and T. Sphicopoulos, 2009. Performance analysis of space time block coding techniques for indoor optical wireless systems. IEEE J. Selected Areas Commun., 27: 1545-1552. DOI: 10.1109/JSAC.2009.091204

Rajbhandari, S., Z. Ghassemlooy and M. Angelova, 2009. Effective denoising and adaptive equalization of indoor optical wireless channel with artificial light using the discrete wavelet transform and artificial neural network. IEEE J. Lightwave Technol., 27: 4493-4500. DOI: 10.1109/JLT.2009.2024432

Rajbhandari, S., Z. Ghassemlooy and M. Angelova, 2011. Wavelet-artificial neural network receiver for indoor optical wireless communications. IEEE J. Lightwave Technol., 29: 2651-2659. DOI: 10.1109/JLT.2011.2162397 